\title{
Revista Colombiana de

\section{Evaluación económica del stent medicado vs. convencional para pacientes con infarto agudo de miocardio con elevación del ST en Colombia}

\section{Mateo Ceballos}

Grupo de Economía de la Salud, Facultad de Ciencias Económicas, Universidad de Antioquia, Medellín, Colombia

Recibido el 1 de noviembre de 2013; aceptado el 16 de junio de 2014

Disponible en Internet el 22 de enero de 2015

\author{
PALABRAS CLAVE \\ Análisis de \\ costo-efectividad; \\ Evaluación \\ económica; \\ Stent; \\ Stents liberadores \\ de fármacos; \\ Infarto agudo de \\ miocardio con \\ elevación del ST
}

\begin{abstract}
Resumen
Objetivo: Analizar la costo-efectividad y el valor esperado de la información perfecta del stent medicado con sirolimus comparado con el convencional para pacientes con infarto agudo de miocardio con elevación del ST en Colombia.

Métodos: Se construyó un modelo de Markov bajo la perspectiva del pagador y un horizonte temporal de diez años. Las probabilidades de transición se extrajeron de estudios clínicos identificados a partir de revisiones de la literatura. Los costos se estimaron mediante el uso de consenso de expertos y manuales tarifarios colombianos. Se realizó un análisis de sensibilidad determinístico alrededor del horizonte temporal, precio del stent medicado y tasa de descuento. Se construyó un análisis de sensibilidad probabilístico (10.000 simulaciones de Monte Carlo) y el valor esperado de la información perfecta para la decisión global y grupos de parámetros.

Resultados: En el caso base, el costo por año de vida ajustado por calidad se ubicó en 53.749 .654 \$. Los resultados no son sensibles al horizonte temporal ni a la tasa de descuento, pero sí al precio del stent medicado. El valor esperado de la información perfecta fue significativamente mayor para la probabilidad de muerte y de sufrir una trombosis muy tardía del stent.

Conclusiones: El stent medicado con sirolimus no es costo-efectivo para pacientes con infarto agudo de miocardio con elevación del ST en Colombia. Se recomienda mayor investigación futura sobre la probabilidad de muerte y trombosis muy tardía del stent, así como en subgrupos específicos de pacientes y stents medicados de segunda generación.

(c) 2013 Sociedad Colombiana de Cardiología y Cirugía Cardiovascular. Publicado por Elsevier España, S.L.U. Todos los derechos reservados.
\end{abstract}




\section{KEYWORDS}

Cost-effectiveness

analysis;

Health economic

evaluation;

Stent;

Drug eluting stent;

ST-elevation

myocardial infarction

\section{Economic evaluation of medicated stent vs. standard stent for patients with acute} myocardial infarction with ST elevation in Colombia

\begin{abstract}
Objective: To perform a cost-effectiveness and value of perfect information analysis comparing sirolimus-eluting stent with bare metal stent for patients with ST-segment elevation myocardial infarction in Colombia.

Methods: We developed a Markov model from the payer perspective and considering a time horizon of 10 years in the base case. Transition probabilities were extracted from clinical studies identified from literature reviews. Costs were estimated using expert consensus and Colombian tariff handbooks. We performed a deterministic analysis around the time horizon, the discount rate and the price of the drug eluting stent. We developed a probabilistic sensitivity analysis $(10,000$ Monte Carlo simulations) and a value of perfect information analysis for the global decision and parameter groups.

Results: In the base case, the cost per quality adjusted life year gained was $\$ 53,749,654$. The results are sensitive to the drug eluting stent price, but not to the time horizon and the discount rate. The expected value of perfect information was significantly higher for the probability of death and very late stent thrombosis.

Conclusions: Sirolimus-eluting stent is not cost-effective for patients with an ST segment elevation myocardial infarction. More future research is recommended around the risk of death and very late stent thrombosis, as well as in specific subgroups of patients and second-generation drug-eluting stents.

(c) 2013 Sociedad Colombiana de Cardiología y Cirugía Cardiovascular. Published by Elsevier España, S.L.U. All rights reserved.
\end{abstract}

\section{Introducción}

Las enfermedades cardiovasculares son la principal causa de muerte en el mundo. En 2008 cobraron la vida de 17 millones de personas, lo que significa un $30 \%$ de todas las defunciones registradas ${ }^{1}$. En Colombia se presenta la misma tendencia, pues estas enfermedades representaron el $28 \%$ de todas las muertes en el año en mención, con tasas de mortalidad de 205,9 y 166,7 por 100.000 habitantes de ambos sexos, respectivamente ${ }^{2}$. Adicionalmente, las enfermedades cardiovasculares se ubican dentro de las primeras diez causas que más años de vida ajustados por discapacidad perdidos generan en Colombia, con tasas que pueden ascender a 104,5 por 1.000 habitantes según la edad ${ }^{3}$. Finalmente, su alta incidencia y prevalencia han conllevado altos y crecientes costos de atención, rehabilitación y seguimiento para los sistemas de salud ${ }^{4}$.

Dentro de las enfermedades cardiovasculares figura el síndrome coronario agudo, asociado con un conjunto de síntomas clínicos compatibles con isquemia miocárdica aguda incluidos cualquier tipo de infarto agudo y angina inestable. De estos el infarto agudo de miocardio con elevación del ST (IAMST), implica en la mayoría de los casos una oclusión completa de la luz de las arterias y habitualmente debe orientarse hacia la revascularización temprana de la arteria comprometida mediante fibrinólisis o intervención coronaria percutánea. En esta última opción es común la implantación de stents medicados o convencionales ${ }^{5}$.

La evidencia respecto a los beneficios del stent medicado no es concluyente en pacientes con IAMST. Pese a que a corto plazo puede reducir la tasa de revascularización del vaso tratado (sin diferencias en muerte, reinfarto o trombosis del stent $)^{6,7}$, a largo plazo puede aumentar la probabilidad de sufrir una trombosis muy tardía del stent ${ }^{8-10}$, la cual ocurre después del primer año de la intervención coronaria percutánea ${ }^{11}$.

Lo anterior plantea una disyuntiva acerca de cuál alternativa debería implementarse en el sistema de salud colombiano con el fin de elegir la estrategia que ofrezca los mayores beneficios en salud, considerando las restricciones presupuestales del país. Así, el objetivo de este trabajo es realizar una evaluación económica, incluyendo un análisis de costo-efectividad y del valor esperado de la información perfecta, comparando el stent medicado y el convencional en pacientes con IAMST en Colombia.

\section{Métodos}

\section{Análisis de costo-efectividad}

Siguiendo los lineamientos de la guía metodológica colombiana ${ }^{12}$, se planteó un análisis de costo-efectividad desde la perspectiva del pagador. Se asumió un horizonte temporal base de diez años y una tasa de descuento del $3 \%$ para costos y efectividad, como lo recomienda dicha guía ${ }^{12}$. La población elegida fueron pacientes mayores de 18 años con IAMST sometidos a una intervención coronaria percutánea. Se consideró únicamente sirolimus como medicamento del stent medicado, debido a que es el que cuenta con mayor evidencia. No se incluyeron en el análisis los stents de segunda generación (everolimus y zotarolimus) debido a 


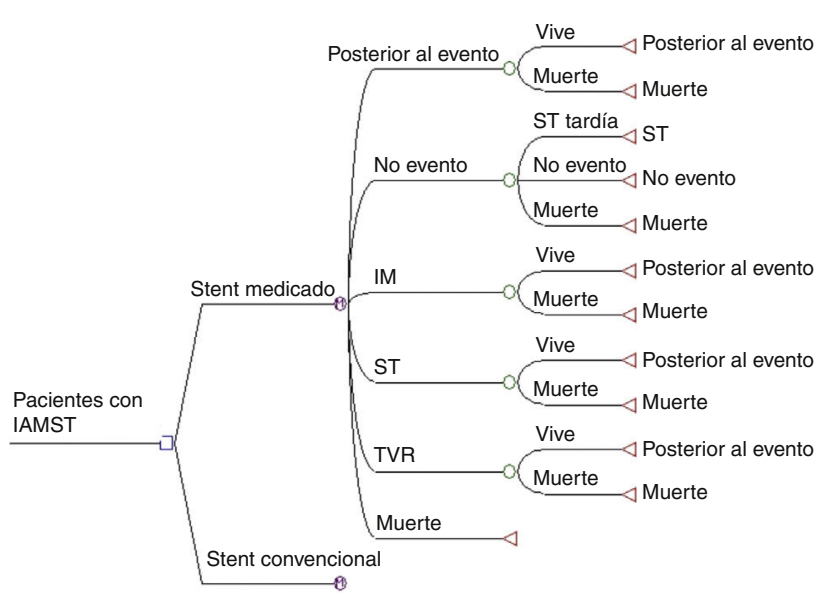

Figura 1 Modelo de Markov.
Tabla 1 Protocolo de búsqueda de la literatura clínica

Base de datos de Cochrane

((Acute Coronary Syndrome[Mesh] OR Acute Coronar*[tiab]

OR Coronary Syndrom*[tiab] OR STEMI[tiab]) AND

(systematic review[tiab] OR Meta-Analysis[pt] OR

Meta-Analysis as Topic[mh] OR Meta-Analys*[tiab] OR

«Cochrane Database Syst Rev»[Journal:__jrid21711])) AND

(Stents[Mesh] OR Stent*[tiab] OR bare metal[tiab] OR

drug elut*[tiab] OR drug coat*[tiab])

PubMed

(STEMI OR ST-segment elevation myocardial infarction) AND

(systematic review OR Meta-Analysis OR Meta-Analys*)

AND (Drug Eluting Stent OR Drug-Coated OR Sirolimus

Eluting Stent) AND (Bare Metal Stent) AND («2010»[Date Publication]: «2013»[Date - Publication]) que no se encontró evidencia clínica suficiente para realizar un modelo de largo plazo que incluyera los principales costos y consecuencias de ambas alternativas.

Para estimar los costos y efectividad de las alternativas se planteó un modelo de Markov siguiendo los principales hallazgos de la literatura clínica, el cual fue validado y modificado según el criterio de expertos en Medicina Interna y Cardiología. En este se consideran los beneficios clínicos del stent medicado a un año, extrapolando los datos en el largo plazo con un análisis de sobrevida.

En el modelo (fig. 1), el cuadrado representa el nodo de decisión inicial; a su derecha se encuentran las alternativas que se comparan. El círculo con una $\mathrm{M}$ en el centro indica el inicio del proceso de Markov, por lo que las ramas siguientes son los estadios de Markov, y los círculos subsecuentes las transiciones que se efectúan anualmente. Los triángulos indican el estadio para el cual se transita en cada ciclo de Markov.

Específicamente, en el modelo los pacientes pueden no tener ningún evento adverso, sufrir un infarto de miocardio (IM), necesitar una revascularización del vaso tratado (TVR), tener una trombosis del stent (ST) o morir. Los individuos que experimentan IM, TVR o ST pueden fallecer o pasar al estadio posterior al evento. Este último es un estadio túnel en el que llegan los pacientes que sufrieron un evento adverso no fatal (IM, TVR, ST) en el largo plazo, por lo que al inicio del primer ciclo de Markov ningún paciente se ubica en él. Adicionalmente, los pacientes que en el primer año no experimentaron ningún evento adverso, pueden sufrir una trombosis muy tardía del stent en el largo plazo y necesitar una nueva intervención, por lo que pasan al estadio ST, en donde fallecen o transitan al estadio posterior al evento.

Las probabilidades de los eventos clínicos se extrajeron de una revisión sistemática de la literatura clínica en la base de datos Cochrane en marzo de 2012, realizada por el grupo desarrollador de la guía nacional de atención integral de pacientes con síndrome coronario agudo ${ }^{13}$. Adicionalmente, con el fin de capturar nueva evidencia se realizó una búsqueda en PubMed en mayo de 2013, restringiéndola a trabajos publicados después de 2010. Los protocolos de ambas búsquedas se presentan en la tabla 1 . Solo se tuvieron en cuenta metaanálisis o revisiones sistemáticas de ensayos clínicos aleatorios que compararan el stent convencional con el medicado con sirolimus. En cuanto a estudios que analizaran diferentes tipos de medicamentos se tuvieron en cuenta únicamente aquellos que presentaran los datos para sirolimus por separado y pudieran extraerse.

A partir de las búsquedas, como base para calcular las probabilidades se seleccionó una revisión de Cochrane que realizó un metaanálisis de 47 ensayos clínicos aleatorios, presentando los resultados a diferentes horizontes temporales, medicamentos y subgrupos de pacientes ${ }^{14}$. La probabilidad de muerte en el largo plazo (ajustada por edad) se estimó con base en las estadísticas vitales del Departamento Administrativo Nacional de Estadística disponibles en su página web. El riesgo relativo de muerte al año utilizado fue de 6,95 y el de largo plazo de 2,63, datos extraídos del trabajo realizado por AstraZeneca para el Instituto Nacional de Excelencia Clínica del Reino Unido ${ }^{15}$, el cual los calculó con información del proyecto nacional de auditoría de isquemia miocárdica del Reino Unido. La probabilidad de sufrir una trombosis muy tardía del stent se extrajo de un metaanálisis con un periodo de seguimiento de tres años ${ }^{16}$. Los datos utilizados corresponden a la probabilidad acumulada de sufrir una trombosis del stent después del primer año de la intervención coronaria.

Las ponderaciones de utilidad para la construcción de los años de vida ajustados por calidad se tomaron de los datos calculados por el estudio PLATO HECON, y reportados en el estudio realizado por AstraZeneca ${ }^{15}$. El dato empleado corresponde al promedio general de todos los países, pues aunque en este estudio participaron pacientes colombianos, los datos no se encontraron desagregados. Se supuso que la utilidad de los estadios IM, ST y TVR es la misma, debido a que solo se cuenta con la información para IM, y los procedimientos y calidad de vida de un paciente que sufre estos eventos son muy similares. Tanto para las probabilidades como para las ponderaciones de utilidad se asumió una distribución beta, siguiendo las recomendaciones de la literatura ${ }^{17}$ (tabla 2).

La medición e identificación de los recursos consumidos se hizo mediante la construcción de un caso típico a partir de la revisión de un protocolo de práctica clínica de un hospital universitario de Medellín, el cual fue validado y modificado según un consenso de especialistas en Medicina Interna y Cardiología. 
Tabla 2 Información de efectividad utilizada en el modelo

\begin{tabular}{lll}
\hline Alternativa & Variable & Valor (parámetro usado para la distribución) \\
\hline Stent medicado & Probabilidad de IM & $0,0333(\mathrm{n}=23 ; \mathrm{N}=690)$ \\
& Probabilidad de ST & $0,0306(\mathrm{n}=23 ; \mathrm{N}=752)$ \\
& Probabilidad de TVR & $0,049(\mathrm{n}=45 ; \mathrm{N}=910)$ \\
& Probabilidad de muerte & $0,0286(\mathrm{n}=26 ; \mathrm{N}=910)$ \\
Stent convencional & Probabilidad de ST tardía & $0,022(\mathrm{n}=26 ; \mathrm{N}=1.187)$ \\
& Probabilidad de IM & $0,048(\mathrm{n}=33 ; \mathrm{N}=687)$ \\
& Probabilidad de ST & $0,039(\mathrm{n}=26 ; \mathrm{N}=659)$ \\
& Probabilidad de TVR & $0,136(\mathrm{n}=124 ; \mathrm{N}=909)$ \\
Ponderaciones de utilidad & Probabilidad de muerte & $0,039(\mathrm{n}=35 ; \mathrm{N}=901)$ \\
& Probabilidad de ST tardía & $0,008(\mathrm{n}=9 ; \mathrm{N}=1.177)$ \\
& No evento & $0,84(\alpha=28.004,078 ; \beta=5.254,922)$ \\
& IM & $0,786(\alpha=1.341,118 ; \beta=380,471)$ \\
\hline
\end{tabular}

IM: infarto de miocardio; ST: trombosis del stent; TVR: revascularización del vaso tratado.

El precio de los stents se dedujo a partir de la información consignada en un centro de referencia de cuarto nivel de Medellín, el cual tiene una alta representatividad en la atención de enfermedades cardiovasculares en el país. Para calcular el rango de precios utilizado en el análisis de sensibilidad y el número de stents por paciente se utilizó una revisión sistemática de la literatura en evaluación económica realizada en la guía de práctica clínica para síndrome coronario agudo ${ }^{13}$. De cada estudio identificado se extrajeron los datos requeridos y se promediaron con el fin de calcular un rango que reflejara los precios máximos y mínimos de las evaluaciones económicas realizadas hasta el momento (tabla 3).

Para la valoración de los procedimientos se usó el manual tarifario del Instituto de Seguros Sociales -ISS- 2001. Para los costos se seleccionó una distribución triangular, en donde se consideró un valor promedio de la tarifa ISS más un 30\%, un mínimo de más $25 \%$ y un máximo de más del $48 \%$. Estos porcentajes fueron calculados por la Alianza Cinets a partir de una encuesta a varias aseguradoras colombianas, que concentraban el $72 \%$ de la población afiliada. Los precios de los insumos se extrajeron de la información suministrada por un hospital de tercer nivel de Medellín.

Para la valoración de los medicamentos se consideró el Sistema de Información de Precios y Medicamentos -SISMEDdel año 2010 canal institucional-laboratorio. Los precios de cada presentación de los medicamentos fueron ponderados por su participación en el mercado, los cuales comprenden tanto los medicamentos genéricos como las moléculas originales. Debido a que los estadios IM, ST y TVR son muy

Tabla 3 Precio unitario y número de stents por paciente

\begin{tabular}{llll}
\hline & Valor mínimo & Valor promedio & Valor máximo \\
\hline $\begin{array}{c}\text { Stent } \\
\text { medicado }\end{array}$ & $2.685 .000 \$$ & $4.485 .000 \$$ & $6.285 .000 \$$ \\
$\begin{array}{c}\text { Stent con- } \\
\text { vencional }\end{array}$ & NA & $818.800 \$$ & NA \\
$\begin{array}{c}\text { Stents por } \\
\text { paciente }\end{array}$ & NA & 1,4 & NA \\
\hline NA: no aplica. & & \\
\end{tabular}

similares en términos de recursos consumidos, se supuso que implican el mismo costo, asociado a la necesidad de una nueva intervención coronaria y al programa de rehabilitación posterior (tabla 4).

Como regla de decisión se utilizó la relación incremental de costo-efectividad (RICE), la cual se comparó con un umbral en caso de no dominancia. Aunque no existe un consenso sobre su definición, se siguió la recomendación de la Organización Mundial de la Salud ${ }^{18}$, que indica fijar un umbral de máximo tres veces el PIB per cápita del país, que para 2011 fue de 40.117.212. \$

Para tener en cuenta la incertidumbre individual en los resultados, se realizó un análisis de sensibilidad determinístico alrededor del precio del stent medicado, el horizonte temporal y la tasa de descuento. Adicionalmente, se desarrolló un análisis de sensibilidad probabilístico (10.000 simulaciones de Monte Carlo), presentando los resultados a través de curvas de aceptabilidad.

\section{Análisis del valor esperado de la información perfecta}

El análisis de costo-efectividad permite resolver la pregunta sobre la adopción de una alternativa con base en la

Tabla 4 Costo del procedimiento y la rehabilitación

\begin{tabular}{|c|c|c|c|}
\hline & $\begin{array}{l}\text { Valor mínimo } \\
\text { (tarifas } \\
\text { ISS+25\%) }\end{array}$ & $\begin{array}{l}\text { Valor } \\
\text { promedio } \\
\text { (tarifas } \\
\text { ISS+30\%) }\end{array}$ & $\begin{array}{l}\text { Valor máximo } \\
\text { (tarifas } \\
\text { ISS+48\%) }\end{array}$ \\
\hline $\begin{array}{l}\text { Costo de la } \\
\text { intervención } \\
\text { coronaria }\end{array}$ & $7.640 .838 \$$ & $7.788 .845 \$$ & $8.321 .670 \$$ \\
\hline $\begin{array}{l}\text { Costo del } \\
\text { programa de } \\
\text { rehabilita- } \\
\text { ción } \\
\text { cardiaca }\end{array}$ & $815.906 \$$ & $848.543 \$$ & $966.033 \$$ \\
\hline
\end{tabular}

ISS: Instituto de seguros sociales. 
mejor evidencia disponible. Sin embargo, debido a la incertidumbre en la estimación del modelo y en los parámetros, existe una posibilidad de tomar una decisión equivocada; es decir, una probabilidad de que una alternativa no elegida sea costo-efectiva si la incertidumbre actual se resolviera. El valor esperado de la información perfecta (VEIP) puede ser interpretado como el costo de la incertidumbre y se entiende como un límite máximo sobre el que la sociedad estaría dispuesta a pagar por mayor investigación futura en el tema.

Aquí es necesario realizar una transformación lineal de la RICE para convertirla en el beneficio neto (BN), una nueva regla de decisión que permite superar algunas de sus limitaciones, y en donde se considera como costo-efectiva la alternativa que tenga el mayor $\mathrm{BN}^{19}$. Para determinar el VEIP, se siguió un modelo no paramétrico introducido por Briggs ${ }^{20}$, el cual puede deducirse directamente del modelo de Markov del apartado anterior y se define como la diferencia entre el BN esperado con información perfecta y con información actual para $j$ alternativas y $\theta$ parámetros inciertos:

$V E I P=E_{\theta} \max _{j} B N(j, \theta)-\max _{j} E_{\theta} B N(j, \theta)$.

Al considerar el BN con información perfecta se asume que se sabría cuál sería el valor de $\theta$ antes de tomar la decisión. Sin embargo, los verdaderos valores de $\theta$ son desconocidos a priori, por lo que su cálculo se encuentra al determinar el valor esperado del mayor BN de las $j$ alternativas en cada simulación del modelo.

El VEIP definido de esta manera considera el costo de la incertidumbre para un solo paciente, por lo que es útil cuantificar los efectos para todos los pacientes que se benefician por el aumento de la información. Esto requiere establecer una población para la cual las alternativas van dirigidas $\left(I_{t}\right)$, especificar el periodo en el cual la información sobre la decisión será útil $(T)$ y descontar los efectos futuros a una tasa $r$. El VEIP para toda la población de pacientes se define como:

VEIP para toda la población $=\operatorname{VEIP} * \sum_{t=1}^{T} I_{t} /(1+r)^{t}$

En este trabajo se asumió la misma tasa de descuento $(r)$ y horizonte temporal $(T)$ utilizado en el análisis de costoefectividad. La población de pacientes $\left(I_{t}\right)$ se extrajo de un estudio de costos de la enfermedad en Colombia ${ }^{21}$, el cual calculó que para 2011 se esperarían 61.512 casos de síndrome coronario agudo, de los cuales se asumió que un 30\% tenía IAMST. Este dato se extrajo del registro global GRACE, un estudio prospectivo mundial en donde se enrolaron 31.982 pacientes de todo el mundo para los cuales se capturaron diferentes características clínicas y económicas $^{22}$. Así, aplicando la fórmula anterior, el multiplicador del VEIP por persona usado fue de 162.135.

Adicionalmente, se calculó el valor esperado de la información perfecta para parámetros, el cual determina el costo de la incertidumbre respecto a uno o varios parámetros; ello permite saber qué tipo de investigación futura sería más valiosa que otra. El VEIPP sigue la misma lógica que el VEIP, pero ahora se está interesado en determinar el valor de la

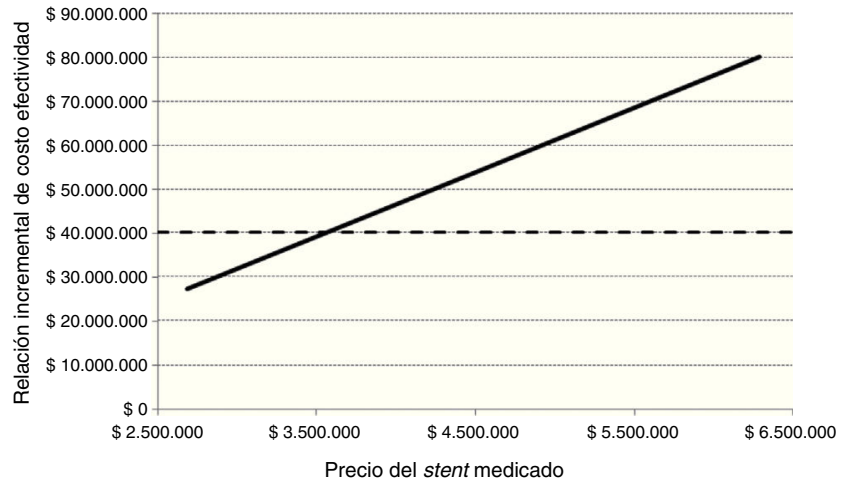

Figura 2 Análisis de sensibilidad: precio del stent medicado.

información perfecta para una parte de todas las variables inciertas.

Siguiendo con el modelo presentado por Briggs ${ }^{20}$, si se está interesado en uno o varios parámetros $\phi$ (parte de todos los $\theta$ inciertos), se debe mantener el resto $(\Psi)$ con la incertidumbre actual. Así, el VEIPP se calcularía como la diferencia en el $\mathrm{BN}$ con información actual y con información perfecta solo para $\phi$ :

$V E I P P_{\phi}=E_{\phi} \max _{j} E_{\Psi / \phi} B N(j, \Psi, \phi)-\max _{j} E_{\theta} B N(j, \Psi, \phi)$

Con información perfecta se sabría qué valores tomarían $\phi$ antes de tomar la decisión, manteniendo la incertidumbre para $\Psi$. Lo anterior implica realizar una simulación interna (inner loop) en donde para cada realización de $\phi$, se calcula el valor esperado del $\mathrm{BN}$ con $\Psi$ parámetros inciertos. Sin embargo, dado que no se conoce a priori $\phi$, se debe hacer una simulación externa (outer loop) para determinar cuáles valores esperados tomará cada realización de estos parámetros.

Con base en algunas recomendaciones de la literatura ${ }^{23}$, para las probabilidades de transición se realizaron 10.000 simulaciones externas y 5.000 internas, mientras que para los costos y las utilidades se asumieron 5.000 externas y 10.000 internas. El cálculo del VEIPP para toda la población sigue la misma lógica que para el VEIP y se usó el mismo multiplicador por persona. Todos los cálculos del análisis de costo-efectividad y del valor esperado de la información perfecta fueron realizados en el software TreeAge Pro $2009^{\circledR}$.

\section{Resultados}

Para el horizonte temporal base de diez años, el stent medicado genera un costo adicional de 5.812.367 $\$$ y un incremento de 0,10814 años de vida ajustados por calidad por paciente, lo cual implica una RICE 53.749.654 \$. Lo anterior indica que el stent medicado no es costo-efectivo, ya que la RICE es superior al umbral, el cual se definió en 40.117.212 \$. Este resultado no es sensible ante modificaciones en el horizonte temporal (tabla 5).

La RICE no varía significativamente con la modificación de la tasa de descuento (datos no mostrados), pero sí con el precio del stent medicado. En la figura 2 la línea horizontal punteada indica el umbral, por lo que si la RICE se ubica por 
Tabla 5 Análisis de sensibilidad: horizonte temporal

\begin{tabular}{lllllll}
\hline $\begin{array}{l}\text { Horizonte } \\
\text { temporal }\end{array}$ & Estrategia & Costo & Costo incremental & AVAC & AVAC incremental & RICE \\
\hline 1 año & Stent convencional & $3.169 .710 \$$ & & 1,55630 & & $149.231 .134 \$$ \\
& Stent medicado & $7.551 .004 \$$ & $4.381 .294 \$$ & 1,58566 & 0,02936 & \\
5 años & Stent convencional & $3.362 .735 \$$ & & 4,29822 & & \\
& Stent medicado & $8.492 .743 \$$ & $5.130 .008 \$$ & 4.37334 & 0,0751 & \\
10 años & Stent convencional & $3.553 .852 \$$ & & 7,08675 & & $5.306 .059 \$$ \\
& Stent medicado & $9.366 .219 \$$ & $5.812 .367 \$$ & 7,19489 & 0,10814 & $53.749 .654 \$$ \\
& Stent convencional & $3.794 .979 \$$ & & 10,7256 & & \\
& Stent medicado & $10.359 .791 \$$ & $6.564 .812 \$$ & 10,8483 & 0,12267 & $53.513 .983 \$$ \\
\hline
\end{tabular}

AVAC: años de vida ajustados por calidad; RICE: relación incremental de costo-efectividad.

encima de esta, el stent medicado no es costo-efectivo. En la figura se observa que para que el stent medicado sea costo efectivo, su precio debe ser igual o menor a 3.580.000 \$.

Los resultados del análisis de sensibilidad probabilístico se presentan en la figura 3, en donde se observa que para el umbral considerado (representado por la línea vertical), la probabilidad de que el stent medicado sea costo-efectivo es del $29 \%$. A partir de un umbral de 54.000.000 \$, el stent medicado obtiene una probabilidad mayor de ser costo efectivo respecto al convencional.

Para el umbral considerado, el VEIP para toda la población de pacientes con IAMST se ubica en 83.789 \$ millones de pesos, alcanzado un valor máximo de 231.330 \$ millones de pesos a una disposición a pagar de 54.000.000 \$ (fig. 4). Adicionalmente, para un umbral de tres veces el PIB per cápita, se encontró un VEIPP cercano a cero para las variables de costos, mientras que para las utilidades se ubicó en 9.679 $\$$ millones de pesos, para las probabilidades conjuntas de TVR, IM y ST de $15.775 \$$ millones de pesos y para la probabilidad de trombosis muy tardía del stent de $19.476 \$$ millones de pesos. El VEIPP más elevado se relacionó con la probabilidad de muerte, que ascendió a 71.114 $\$$ millones de pesos (fig. 5).

Los valores más altos del VEIPP se ubicaron en un umbral de 54.000.000 \$, alcanzando valores de $512 \$$ millones de pesos para costos, de $45.644 \$$ millones de pesos para las probabilidades conjuntas de TVR, IM y ST, de 21.477 \$ millones de pesos para las utilidades, de $60.060 \$$ millones de pesos

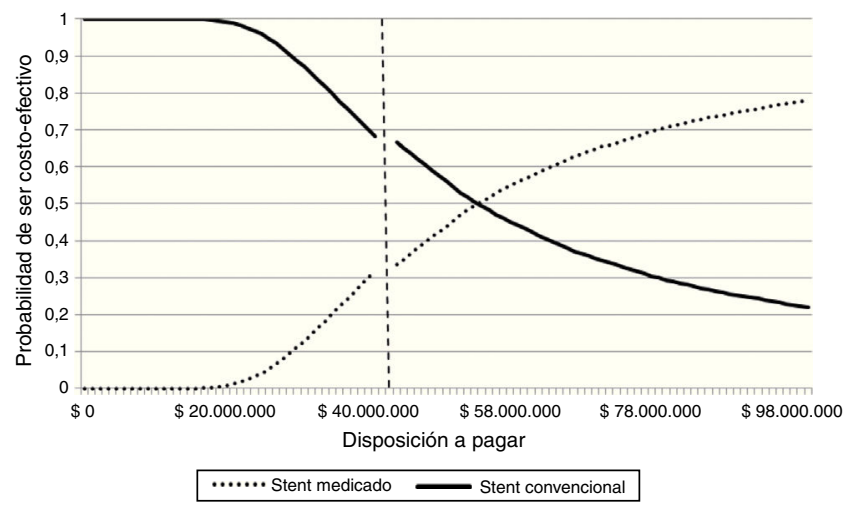

Figura 3 Curva de aceptabilidad.

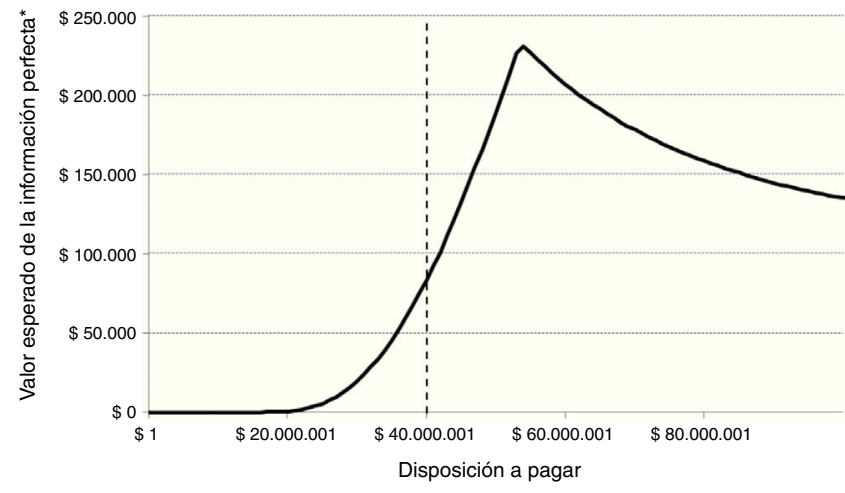

*Valores en millones de pesos.

Figura 4 Valor esperado de la información perfecta.

para la probabilidad de trombosis muy tardía del stent y de 219.770 \$ millones de pesos para la probabilidad de muerte.

\section{Discusión}

Los resultados de este análisis económico sugieren que en Colombia el stent medicado no es costo-efectivo para

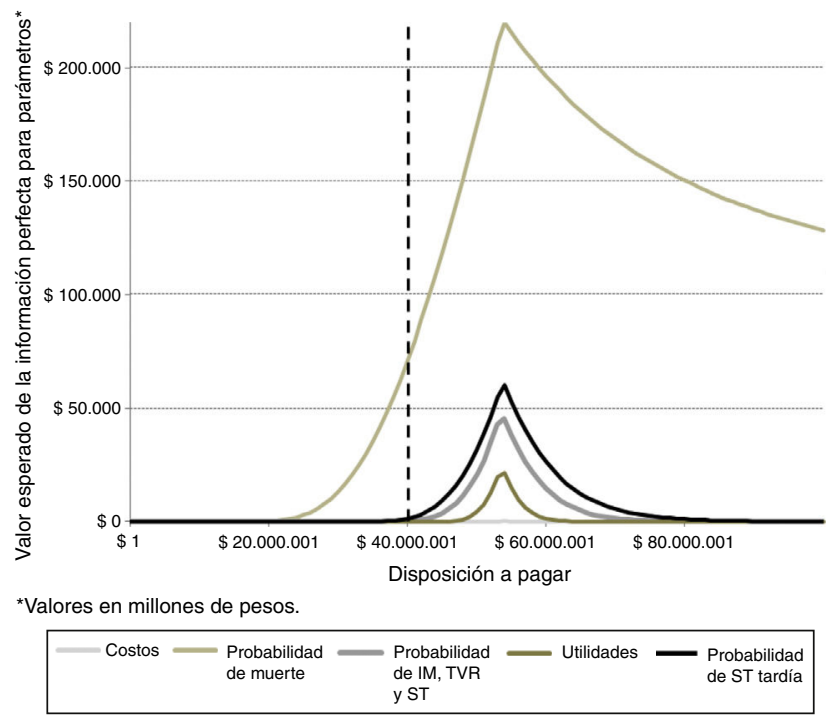

Figura 5 Valor esperado de la información perfecta para parámetros. 
pacientes con IAMST, incluso en un horizonte temporal de largo plazo. Lo anterior se debe al alto diferencial de precios, y el hecho de que los beneficios clínicos que genera el stent medicado en la reducción de la tasa de revascularización del vaso tratado en el primer año y siguientes, se ven compensados por el aumento en la probabilidad de sufrir una trombosis muy tardía del stent a largo plazo.

En el análisis de sensibilidad se evidencia que el precio del stent medicado es una variable que influye significativamente en los resultados. El diferencial de precios entre los dos tipos de stent es una variable clave que el decisor debe tener en cuenta, y las políticas regulatorias podrían ayudarle a determinar la deseabilidad de uno u otro tipo de stent para el sistema de salud.

Por otro lado, se encuentra un VEIP relativamente alto, lo cual indica que el costo de la incertidumbre en la decisión es significativo. La fuente de dicha incertidumbre puede deberse a que la RICE está relativamente cerca del umbral. Adicionalmente, el VEIPP resultó más elevado para la probabilidad de muerte y de trombosis muy tardía del stent, lo que sugiere que podría ser costo-efectiva, si bien se requiere mayor investigación futura alrededor de estos desenlaces. Sin embargo, es importante notar que lo anterior depende de los costos esperados de la realización de mayor investigación, los cuales no fueron calculados en este trabajo.

Los hallazgos de esta investigación van en la misma línea que lo hallado en otras evaluaciones económicas para pacientes con IAMST, las cuales han encontrado resultados poco favorables para el stent medicado, y mucha incertidumbre alrededor de la estimación y la decisión, recomendando, por tanto, mayor investigación respecto al tema ${ }^{24,25}$. No obstante, es importante notar que hacer una comparación directa entre los resultados de la literatura internacional puede no ser adecuado debido a que las diferencias en aspectos metodológicos y en los modelos tarifarios y de contratación pueden ser muy variables, e impactar de manera significativa los resultados encontrados según el contexto de salud ${ }^{26}$.

En este trabajo se realizó una evaluación económica de novo con base en la mejor evidencia disponible para el país, e incluyendo un análisis del VEIP, el cual no ha sido incorporado con frecuencia en las evaluaciones económicas hechas en Colombia. El VEIP se ha perfilado como un elemento fundamental para la elaboración de un marco de toma de decisiones racional en los sistemas de salud ${ }^{27}$, y es un insumo adicional al análisis de costo-efectividad que enriquece el alcance de la evaluación económica.

El trabajo tuvo algunas limitaciones. En primer lugar, aunque el estudio que calculó las ponderaciones de utilidad incluyó pacientes colombianos, estas se calcularon con base en valoraciones de la población del Reino Unido, las cuales pueden no ser aplicables a nuestro medio. En este sentido es necesario que en el país se avance en la estimación de ponderaciones de utilidad propias. En segundo lugar, la revisión de Cochrane utilizó datos no publicados, y hace hincapié en cierta falta de estandarización de los diseños y definiciones por parte de los ensayos clínicos aleatorios incluidos. En tercer lugar, en el modelo se excluyó la posibilidad de tratar a los pacientes con bypass o trombólisis en sucesivas intervenciones. Por último, en el análisis no se incluyeron subgrupos de pacientes específicos con IAMST (diabéticos, con un tamaño de vaso pequeño, entre otros) debido a que no se encontró suficiente evidencia clínica para construir el modelo diferenciado para cada subgrupo. Además, tampoco se consideraron dentro del modelo los posibles costos y efectividad de los stent medicados de segunda generación (everolimus y zotarolimus), los cuales han aumentado progresivamente su participación en la práctica clínica actual. Los dos puntos anteriores se identifican como posibles focos de investigación futura en el tema, que permitirían generar un mayor flujo de información basado en evidencia científica para los tomadores de decisiones del país.

\section{Conclusiones}

Los resultados obtenidos en este trabajo indican que el stent medicado con sirolimus no es costo-efectivo para pacientes con IAMST en Colombia. Lo anterior se debe a que la reducción en la probabilidad de la tasa de revascularización del vaso tratado con el stent medicado se ve compensada por un aumento en la probabilidad de sufrir una trombosis muy tardía del stent. Variables como el diferencial de precios entre los dos tipos de stent, y la probabilidad de muerte y de trombosis muy tardía del stent, determinan su deseabilidad para el sistema de salud colombiano. Se recomienda mayor investigación alrededor de estos desenlaces clave, así como en subgrupos específicos de pacientes con IAMST. También se identifica la necesidad de realizar estudios económicos adicionales que consideren los costos y efectividad de los stents de segunda generación (everolimus y zotarolimus), los cuales están usándose cada vez más en la práctica clínica.

\section{Nota}

Los resultados de esta investigación son parte del trabajo de grado llevado a cabo por el autor para optar por el título de Economista en la Universidad de Antioquia. Parte de la información empleada en el trabajo fue extraída de la Guía de Atención Integral basada en la evidencia, para la detección temprana, atención integral, seguimiento y rehabilitación de pacientes con diagnóstico de un evento coronario agudo, realizada por la Universidad de Antioquia en el marco de la Alianza Cinets, y financiada por Colciencias y el Ministerio de Salud y Protección Social, a partir de la convocatoria 500 de 2009.

\section{Conflicto de intereses}

El autor declara no tener ningún conflicto de intereses.

\section{Agradecimientos}

A los doctores Aurelio Mejía y Juan David Ramírez por su acompañamiento y dedicación. A los Drs. Juan Manuel Senior, Natalia Acosta y a los demás autores de la guía por los aportes hechos a esta investigación.

\section{Bibliografía}

1. Organización Mundial de la Salud. Global Status Report on Noncommunicable Diseases, 2010-2011. [consultado 5 May 
2014]. Disponible en: http://whqlibdoc.who.int/publications/ 2011/9789240686458_eng.pdf

2. Organización Mundial de la Salud. Noncommunicable diseases country profiles 2011. 2011. [consultado 5 May 2014]. Disponible en: http://whqlibdoc.who.int/publications/2011/ 9789241502283_eng.pdf

3. Bolívar-Mejía A, Vesga-Angarita BE. Burden of cardiovascular disease in Colombia. 2013. En: Alfonso R. Current topics in public health. ISBN 978-953-51-1121-4, 732 páginas.

4. Heidenreich PA, Trogdon JG, Khavjou OA, Butler J, Dracup K, Ezekowitz MD, et al. Forecasting the future of cardiovascular disease in the United States: a policy statement from the American Heart Association. Circulation. 2011;123(8):933-44.

5. Cassiani CA, Cabrera A. Síndromes coronarios agudos: epidemiología y diagnóstico. Salud Uninorte. 2009;25(1):118-34.

6. Dibra A, Tiroch K, Schulz S, Kelbaek H, Spaulding C, Laarman GJ, et al. Drug-eluting stents in acute myocardial infarction: updated meta-analysis of randomized trials. Clin Res Cardiol. 2010;99(6):345-57.

7. Hao PP, Chen YG, Wang XL, Zhang Y. Efficacy and safety of drug-eluting stents in patients with acute ST-segment-elevation myocardial infarction: a meta-analysis of randomized controlled trials. Tex Heart Inst J. 2010;37(5):516-24.

8. De Luca G, Dirksen MT, Spaulding C, Kelbaek H, Schalij M, Thuesen L, et al. Drug-eluting vs. bare-metal stents in primary angioplasty: a pooled patient-level meta-analysis of randomized trials. Arch Intern Med. 2012;172(8):611-21.

9. Kalesan B, Pilgrim T, Heinimann K, Räber L, Stefanini GG, Valgimigli $\mathrm{M}$, et al. Comparison of drug-eluting stents with bare metal stents in patients with ST-segment elevation myocardial infarction. Eur Heart J. 2012;33(8):977-87.

10. Sethi A, Bahekar A, Bhuriya R, Bajaj A, Kovacs D, Ahmed A, et al. Drug-eluting stents versus bare metal stents in ST elevation myocardial infarction at a follow-up of three years or longer: A meta-analysis of randomized trials. Exp Clin Cardiol. 2012;17(4):169-74.

11. Cutlip DE, Windecker S, Mehran R, Boam A, Cohen DJ, van Es $\mathrm{GA}$, et al. Clinical end points in coronary stent trials: a case for standardized definitions. Circulation. 2007;115(17):2344-51.

12. Ministerio de la Protección Social, Colciencias, Centro de Estudios e Investigación en Salud de la Fundación Santa Fe de Bogotá, Escuela de Salud Pública de la Universidad de Harvard. Guía Metodológica para el desarrollo de Guías de Atención Integral en el Sistema General de Seguridad Social en Salud Colombiano. Bogotá: Colciencias; 2010.

13. Ministerio de Salud y Protección Social, Colciencias, Universidad de Antioquia. Guía de práctica clínica para el síndrome coronario agudo. Bogotá: GPC-SCA; 2013.

14. Greenhalgh J, Hockenhull J, Rao N, Dundar Y, Dickson RC, Bagust A. Drug-eluting stents versus bare metal stents for angina or acute coronary syndromes. Cochrane Database Syst Rev. 2010;5:CD004587.

15. AstraZeneca. Ticagrelor for the treatment of Acute Coronary Syndromes. National Institute of Clinical Excellence. United Kingdom. 2010. [consultado 8 Jun 2013]. Disponible en: http:// www.nice.org.uk/nicemedia/live/12169/55171/55171.pdf

16. Piccolo R, Cassese S, Galasso G, Niglio T, De Rosa R, De Biase C, et al. Long-term clinical outcomes following sirolimus-eluting stent implantation in patients with acute myocardial infarction. A meta-analysis of randomized trials. Clin Res Cardiol. 2012;101(11):885-93.

17. Claxton K. Exploring uncertainty in cost-effectiveness analysis. Pharmacoeconomics. 2008;26(9):781-98.

18. Organización Mundial de la Salud. Macroeconomics and Health: Investing in health for economic development. Geneva: Organización Mundial de la Salud; 2001.

19. Stinnett AA, Mullahy J. Net health benefits: a new framework for the analysis of uncertainty in cost-effectiveness analysis. Med Decis Making. 1998;18 2 Suppl:S68-80.

20. Briggs A, Claxton K, Sculpher M. Decision modelling for health economic evaluation. 1.st. ed. New York: Oxford University Press; 2006

21. Castellanos JC. Proyección de costos en Colombia de la atención inicial del síndrome coronario agudo. Univ Med. 2011;52(1):36-43.

22. Goodman SG, Huang W, Yan AT, Budaj A, Kennelly BM, Gore JM, et al. The expanded Global Registry of Acute Coronary Events: baseline characteristics, management practices, and hospital outcomes of patients with acute coronary syndromes. Am Heart J. 2009;158(2):193-201, e1- e5.

23. Brennan A, Kharroubi S, O’hagan A, Chilcott J. Calculating partial expected value of perfect information via Monte Carlo sampling algorithms. Med Decis Making. 2007;27(4):448-70.

24. Canoui-Poitrine F, Jeanblanc G, Alberti C, Armoogum P, Cebrian $A$, Carrié $D$, et al. Cost effectiveness of sirolimus-eluting stents compared with bare metal stents in acute myocardial infarction: insights from the TYPHOON trial. Appl Health Econ Health Policy. 2009;7(1):19-29.

25. Bäumler M, Stargardt T, Schreyögg J, Busse R. Cost effectiveness of drug-eluting stents in acute myocardial infarction patients in Germany: results from administrative data using a propensity score-matching approach. Appl Health Econ Health Policy. 2012;10(4):235-48.

26. Drummond M, Barbieri M, Cook J, Glick HA, Lis J, Malik F, et al. Transferability of economic evaluations across jurisdictions: ISPOR Good Research Practices Task Force report. Value Health. 2009;12(4):409-18.

27. Claxton K, Sculpher M, Drummond M. A rational framework for decision making by the National Institute For Clinical Excellence (NICE). Lancet. 2002;360(9334):711-5. 